4. Huang JS, Donohue M, Golnari G, Fernandez S, et al. Pediatricians' weight assessment and obesity management practices. BMC Pediatr. 2009; 9:19.

5. Barlow SE, Dietz WH, Klish WJ, Trowbridge FL. Medical evaluation of overweight children and adolescents: reports from pediatricians, pediatric nurse practitioners, and registered dieticians. Pediatrics. 2002; 110(1 Pt 2):222-8.

6. Benson L, Baer HJ, Kaelber DC. Trends in the diagnosis of overweight and obesity in children and adolescents: 19992007. Pediatrics. 2009; 23(1):e153-8.

7. Patel AI, Madsen KA, Maselli JH, Cabana M, et al. Under diagnosis of pediatric obesity during outpatient preventive care visits. Acad Pediatr. 2010; 10(6):405-9.

8. Dilley JK, Martin LA, Sullivan C, Seshadri R, et al. Identification of overweight status is associated with higher rates of screening for comorbidities of overweight in pediatric primary care practice. Pediatrics. 2007; 119(1):e148-55.

9. Benson LJ, Baer HJ, Kaelber DC. Screening for obesityrelated complications among obese children and adolescents: 1999-2008. Obesity. 2011; (19):1077-82.

10. Smith AJ, Skow Á, Bodurtha J, Kinra S. Health information technology in screening and treatment of child obesity: a systematic review. Pediatrics. 2013; 131(3):e894-902.
11. World Health Organization Multicentre Growth Reference Study Group. WHO Child Growth Standards: Length/ height-for-age, weight-for-age, weight-for-length, weightfor-height and body mass index-for-age: Methods and development. Geneva, 2006. [Accessed on: November 14 $4^{\text {th }}$, 2018]. Available at: https://www.who.int/childgrowth/ standards/Technical_report.pdf.

12. Walker $\mathrm{O}$, Strong $\overline{\mathrm{M}}$, Atchinson $\mathrm{R}$, Saunders J, et al. A qualitative study of primary care clinicians' views of treating childhood obesity. BMC Fam Pract. 2007; 8:50 AM.

13. Otero P, Duran P, Setton D, Eymann F, et al. Mismatch between the prevalence of overweight and obese children and adolescents and recording in electronichealth records: a cross-sectional study. Inform Prim Care. 2011;19(2):75-82.

14. Cook S, Weitzman M, Auinger P, Barlow SE. Screening and counseling associated with obesity diagnosis in a national survey of ambulatory pediatric visits. Pediatrics. 2005; 116(1):112-6.

15. Keehbauch J, Miguel GS, Drapiza L, Pepe J, et al. Increased documentation and management of pediatric obesity following implementation of an EMR upgrade and education. Clin Pediatr (Phila). 2012; 51(1):31-8.

\title{
Acute kidney injury and diabetic ketoacidosis in pediatric patients: Risk factors
}

\author{
Carlos Sánchez García, M.D. ${ }^{a}$, Mónica Briones Castellanos, M.D. ${ }^{a}$ and Artemio Velasco Morales, M.D. ${ }^{a}$
}

\begin{abstract}
Acute kidney injury is a cause of morbidity in children with diabetes in developing countries, especially in patients with diabetic ketoacidosis. The objective of this study was to identify the risk factors for acute kidney injury in patients with diabetic ketoacidosis. This was a retrospective cohort study. A total of 50 patients with diabetic ketoacidosis were included; $54 \%$ developed kidney injury. These had higher glucose and uric acid levels (541 mg/dL vs. $407 \mathrm{mg} / \mathrm{dL}, p=0.014$ and $8.13 \mathrm{mg} /$ $\mathrm{dL}$ vs. $5.72 \mathrm{mg} / \mathrm{dL}, p=0.015$, respectively). Uric acid levels above $6.5 \mathrm{mg} / \mathrm{dL}$ showed an odds ratio of $6.910(p=0.027)$ for kidney injury. To conclude, hyperuricemia was a risk factor for acute kidney injury in these patients. Prospective studies are required to determine the role of uric acid in the pathogenesis of acute kidney injury in patients with diabetes.

Key words: diabetic ketoacidosis, acute kidney injury, pediatrics, uric acid.
\end{abstract}

a. Hospital Niño de Saltillo. Saltillo, Coahuila, Mexico.

E-mail address:

Carlos Sánchez, M.D.: sanchez.carlos8516@gmail.com

Funding: None.

Conflict of interest: None.

Received: 3-26-2019

Accepted: 9-16-2019 http: / / dx.doi.org/10.5546/ aap.2020.eng.135

To cite: Sánchez García C, Briones Castellanos M, Velasco Morales A. Acute kidney injury and diabetic ketoacidosis in pediatric patients: Risk factors. Arch Argent Pediatr 2020;118(2):135-138.

\section{INTRODUCTION}

Acute kidney injury is defined as an abrupt decrease in kidney function, leading to electrolyte, acid-base, and water imbalances and waste product accumulation. ${ }^{1}$ The incidence of acute kidney injury has increased, especially in critically-ill hospitalized patients. ${ }^{2}$

The presence of diabetes mellitus is a risk factor for acute kidney injury because it increases renal vulnerability to ischemia. ${ }^{3}$ Acute kidney injury is a predictor of morbidity in patients with type 1 diabetes mellitus; even mild episodes are associated with a cumulative risk for chronic kidney disease. ${ }^{4}$

Diabetic ketoacidosis is a common presentation in patients diagnosed with type 1 diabetes mellitus. ${ }^{5}$ It is also the main cause of 
morbidity and mortality in children with diabetes in developing countries. ${ }^{6}$

It is therefore important to recognize acute kidney injury in patients diagnosed with diabetic ketoacidosis. The objective of this study was to identify the risk factors for acute kidney injury in pediatric patients with diabetic ketoacidosis.

\section{METHODOLOGY}

This was a retrospective cohort study. The medical records of patients admitted to the pediatric intensive care unit of Hospital del Niño de Saltillo "Dr. Federico Gómez Santos" between January 2007 and December 2017 were reviewed. Patients aged 1 month to 18 years old and diagnosed with moderate to severe diabetic ketoacidosis were included. Severity classification was based on the criteria by the American Diabetes Association. Patients with pre-existent chronic kidney disease and those with missing data for the diagnosis of acute kidney injury or for the classification of diabetic ketoacidosis severity were excluded from the study. This study was assessed and approved by the hospital's Research Ethics Committee; no informed consent was required.

Acute kidney injury was defined based on the clinical practice guidelines for the staging of acute kidney injury by Kidney Disease: Improving Global Outcomes (KDIGO). The lowest serum creatinine value included in the medical record was defined as baseline creatinine. Baseline creatinine was compared to the highest value recorded during the course of the diabetic ketoacidosis episode.

The patients' clinical characteristics (age, sex, weight, heart rate, systolic and diastolic blood pressure, length of stay) and laboratory values (hematocrit, bicarbonate, sodium, chloride, glucose, and uric acid levels) were compared between patients who developed acute kidney injury and those who did not. Quantitative outcome measures were analyzed using Student's $t$ test, and qualitative outcome measures, using the $\chi^{2}$ test.

Blood pressure, heart rate, and bicarbonate, sodium, chloride, glucose, hematocrit, and uric acid levels were assessed in order to identify risk factors. A univariate logistic regression was done for each outcome measure; those with a $p$ value $\leq 0.25$ were selected for assessment with a multivariate logistic regression to identify independent risk factors.

A value of $p<0.05$ was considered statistically significant. The statistical analysis was done using the IBM SPSS Statistics software (IBM Corp. Released 2013. IBM SPSS Statistics for Windows, Version 21.0. Armonk NY: IBM Corp.).

\section{RESULTS}

A total of 70 patients diagnosed with diabetic ketoacidosis were admitted to the pediatric intensive care unit. Of them, 20 were excluded;

TABLE 1. Characteristics of patients with diabetic ketoacidosis $(N=50)$

\begin{tabular}{lccc}
\hline & Without kidney injury & With kidney injury & $p$ value \\
\hline Male, $\mathrm{n}(\%)$ & $6(37.5)$ & $10(62.5)$ & 0.684 \\
Female, $\mathrm{n}(\%)$ & $17(50)$ & $17(50)$ & \\
Age [years old], mean (SD) & $9.91(4.05)$ & $11.04(3.75)$ & 0.312 \\
Weight [kg], mean (SD) & $34.26(19.47)$ & $39.49(16.45)$ & 0.315 \\
Systolic blood pressure [mmHg], mean (SD) & $110.47(15.04)$ & $109.70(16.81)$ & 0.864 \\
Diastolic blood pressure [mmHg], mean (SD) & $67.26(10.16)$ & $66.11(13.29)$ & 0.731 \\
Baseline creatinine [mg/dL], mean (SD) & $0.49(0.12)$ & $0.52(0.11)$ & 0.354 \\
Bicarbonate level [mmol/L], mean (SD) & $6.43(3.01)$ & $5.40(3.06)$ & 0.248 \\
Sodium level [mmol/L], mean (SD) & $136.76(6.48)$ & $138.79(6.45)$ & 0.286 \\
Chloride level [mmol/L], mean (SD) & $101.25(22.12)$ & $108.35(6.42)$ & 0.190 \\
Glucose level [mg/dL], mean (SD) & $407.65(146.01)$ & $541.62(220.98)$ & 0.014 \\
Hematocrit [\%], mean (SD) & $40.55(4.20)$ & $38.77(4.95)$ & 0.217 \\
Uric acid level [mg/dL], mean (SD) & $5.72(2.23)$ & $8.13(3.63)$ & 0.015 \\
Length of stay [days], mean (SD) & $4.47(1.7)$ & $4.77(1.84)$ & 0.554 \\
\hline
\end{tabular}

SD: standard deviation. 
5 because they did not meet the diagnostic criteria for moderate to severe diabetic ketoacidosis and 15 because their medical records were incomplete.

Fifty patients were included in the analysis; 27 patients developed some degree of acute kidney injury (54\%). Among patients in the acute kidney injury group, mean age was 11 years vs. 9.9 years in the no acute kidney injury group $(p=0.312)$. The average heart rate in the no acute kidney injury group was lower (111.82 vs. 122.58 beats per minute; $p=0.079$ ). No significant differences were observed in systolic and diastolic blood pressure.

Sodium, chloride, bicarbonate, and hematocrit values did not show significant differences between groups. However, glucose and uric acid levels were higher in the group that developed kidney injury; glucose: $541 \mathrm{mg} / \mathrm{dL}$ vs. $407 \mathrm{mg} / \mathrm{dL}$ $(p=0.014)$; uric acid: $8.13 \mathrm{mg} / \mathrm{dL}$ vs. $5.72 \mathrm{mg} / \mathrm{dL}$ $(p=0.015)$. No difference was observed between groups in terms of length of stay (Table 1).

Using a univariate logistic regression, each 10-beat-per-minute increment in the heart rate was associated with a $24 \%$ increase in the probability of developing acute kidney injury $(p=0.092)$. For each $\mathrm{mmol} / \mathrm{L}$ reduction in bicarbonate level, the probability of developing acute kidney injury increased by $12.2 \%(p=0.25)$. For each $\mathrm{mmol} / \mathrm{L}$ increase in chloride levels, the probability of developing acute kidney injury increased by $6.4 \%(p=0.223)$. Likewise, for each $100 \mathrm{mg} / \mathrm{dL}$ of serum glucose, the odds ratio for kidney injury increased by $50 \%(p=0.021)$. In the case of uric acid, the odds ratio for acute kidney injury was $1.302(p=0.031)$. The other outcome measures were not significant in the univariate analysis.

To assess independent risk factors, heart rate and chloride, glucose, and uric acid levels were included in the multivariate analysis. Uric acid levels above $6.5 \mathrm{mg} / \mathrm{dL}$ showed an odds ratio of 6.910 (95\% confidence interval: 1.247-38.298), with a $p$ value $=0.027$. The other outcome measures were not statistically significant (Table 2).

\section{DISCUSSION}

Patients with diabetic ketoacidosis are at risk for acute kidney injury. This condition mostly resolves with the management of ketoacidosis. ${ }^{7}$ Some studies assessing the presentation of kidney injury and its risk factors in these patients have been published.

In a retrospective study conducted in adults, acute kidney injury occurred in $50 \%$ of patients diagnosed with diabetic ketoacidosis. Age and glucose and protein levels were independently associated with its presentation. ${ }^{8}$

In relation to pediatric patients, Hursh et al. reported that $64 \%$ of patients hospitalized with diabetic ketoacidosis developed acute kidney injury. Bicarbonate levels below $10 \mathrm{mmol} / \mathrm{L}$, heart rate, and serum sodium levels above $145 \mathrm{mmol} / \mathrm{L}$ were independent risk factors for kidney injury, unlike in our study. ${ }^{9}$

Recently, in a study published in $2019,30 \%$ of patients with diabetic ketoacidosis developed acute kidney injury. Sodium, glucose, and lactate levels were higher in this group when compared to those who did not develop kidney injury. ${ }^{10}$ In our study, glucose levels were also higher in patients who developed acute kidney injury; however, no significant difference was observed in terms of sodium. Likewise, no difference was observed between groups in relation to the length of stay.

We did not find any study that assessed uric acid levels as a risk factor for acute kidney injury in patients with diabetic ketoacidosis. However, its involvement in other conditions has been studied.

The incidence of acute kidney injury is associated with high uric acid levels at the time of hospitalization. ${ }^{11}$ High preoperative uric acid levels have been correlated to a greater risk for

TABLE 2. Independent risk factors for acute kidney injury in diabetic ketoacidosis

\begin{tabular}{lccc}
\hline & OR & 95 \% CI & $p$ value \\
\hline Heart rate & 1.003 & $(0.966-1.041)$ & 0.881 \\
Serum chloride & 1.118 & $(0.919-1.359)$ & 0.264 \\
Serum glucose & 1.005 & $(0.998-1.011)$ & 0.155 \\
Serum uric acid $>6.5 \mathrm{mg} / \mathrm{dL}$ & 6.910 & $(1.247-38.298)$ & 0.027 \\
\hline
\end{tabular}

OR: odds ratio; CI: confidence interval. 
acute kidney injury in heart surgery. ${ }^{12}$ It has even been proposed that, in patients with burn wounds, the inflammatory process related to uric acid may be a pathogenic factor associated with early kidney failure. ${ }^{13}$

In experimental studies, hyperuricemia affects the self-regulatory response of the kidney, thus triggering hypoperfusion and tubular inflammation. The pathogenesis of acute kidney injury related to uric acid is also mediated by a process that does not involve crystal formation and plays a major role in acute complications. ${ }^{14}$

Pediatric patients with acute kidney injury, regardless of severity, are at a risk for subsequent chronic kidney disease. ${ }^{15}$ Acute kidney injury is a common complication in pediatric patients with diabetic ketoacidosis.

The limitations of this study were its retrospective nature and the small number of patients. Prospective studies with an adequate sample size are required to identify modifiable risk factors and determine the role of uric acid in the pathogenesis of acute kidney injury in patients with diabetes.

\section{CONCLUSIONS}

Hyperuricemia is correlated to the development of acute kidney injury. It is difficult to establish if hyperuricemia is the cause or the result of kidney failure because dehydration and other factors contribute to its presentation. Its role as a risk marker should not be ruled out. No other outcome measure was shown to be an independent risk factor.

\section{REFERENCES}

1. Ciccia E, Devarajan P. Pediatric acute kidney injury: prevalence, impact and management challenges. Int $\mathrm{J}$ Nephrol Renovasc Dis. 2017; 10:77-84.
2. AndreoliSP. Acutekidney injury inchildren. Pediatr Nephrol. 2009; 24(2):253-63.

3. Patschan D, Müller GA. Acute kidney injury in diabetes mellitus. Int J Nephrol. 2016; 2016:6232909.

4. Monseu M, Gand E, Saulnier PJ, RagotS, et al. Acutekidney injury predicts major adverse outcomes in diabetes: synergic impact with low glomerular filtration rate and albuminuria. Diabetes Care. 2015; 38(12):2333-40.

5. Raghupathy P. Diabetic ketoacidosis in children and adolescents. Indian J Endocrinol Metab. 2015; 19(Suppl 1): S55-7.

6. Razavi Z. Frequency of ketoacidosis in newly diagnosed type 1 diabetic children. Oman Med J. 2010; 25(2):114-7.

7. Woodrow G, Brownjohn AM, Turney JH. Acute renal failure in patients with type 1 diabetes mellitus. Postgrad Med J. 1994; 70(821):192-4.

8. Orban JC, Maizière EM, Ghaddab A, Van Obberghen E, et al. Incidence and characteristics of acute kidney injury in severe diabetic ketoacidosis. PloS One. 2014; 9(10):e110925.

9. Hursh BE, Ronsley R, Islam N, Mammen C, et al. Acute kidney injury in children with type 1 diabetes hospitalized for diabeticketoacidosis. JAMA Pediatr. 2017; 171(5):e170020.

10. Weissbach A, Zur N, Kaplan E, Kadmon G, et al. Acute kidney injury in critically ill children admitted to the PICU for diabetic ketoacidosis. A retrospective study. Pediatr Crit Care Med. 2019; 20(1):e10-4.

11. Kaufeld T, Foerster KA, Schilling T, Kielstein JT, et al. Preoperative serum uric acid predicts incident acute kidney injury following cardiac surgery. BMC Nephrol. 2018; 19(1):161.

12. Liang J, Zhang P, Hu X, Zhi L. Elevated serum uric acid after injury correlates with the early acute kidney in severe burns. Burns. 2015; 41(8):1724-31.

13. Sánchez-Lozada LG, Tapia E, Santamaría J, Avila-Casado $\mathrm{C}$ et al. Mild hyperuricemia induces vasoconstriction and maintains glomerular hypertension in normal and remnant kidney rats. Kidney Int. 2005; 67(1):237-47.

14. Ejaz AA, Dass B, Kambhampati G, Ejaz NI, et al. Lowering serum uric acid to prevent acute kidney injury. Med Hypotheses. 2012; 78(6):796-9.

15. Mammen C, Al Abbas A, Skippen P, Nadel H, et al. Longterm risk of CKD in children surviving episodes of acute kidney injury in the intensive care unit: a prospective cohort study. Am J Kidney Dis. 2012; 59(4):523-30. 